\title{
A second order, non-linear elliptic boundary value problem with generalized Goursat data $\left(^{*}\right)$.
}

\author{
by A.K. Azrz(1), R.P. Gilbert ( $\left.{ }^{2}\right)$ and H.C. Howard (3)
}

Summary. - In this paper the case of generalized Goursat data is considered for the non-linear partial differential equation $\Delta u=f\left(x, y, u, u_{x}, u_{y}\right)$. The existence and uniqueness of $a$ solution is demonstrated, under certain conditions, by employing the contraction mapping method in a suitable Banach space.

\section{1. - Introduetion.}

In this paper we show that under certain conditions a unique (complex valued) solution exists for the non-linear elliptio equation

$$
\Delta u=\tilde{f}\left(x, y, u, u_{x}, u_{y}\right)
$$

which satisfies a generalized boundary condition on each of the analytic curves $\Gamma_{1} \equiv\left\{(x, y) \mid y=f_{1}(x), o \leq x \leq a\right\}$ and $\Gamma_{2} \equiv\left\{(x, y) \mid x=f_{2}(y), o \leq y \leq b\right\}$ namely

$$
\begin{aligned}
& u_{x}(x, y)=\tilde{\alpha}_{0}(x) u(x, y)+\tilde{\alpha}_{1}(x) u_{y}(x, y)+\tilde{g}(x) \text { on } \Gamma_{1} \\
& u_{y}(x, y)=\tilde{\beta}_{0}(y) u(x, y)+\tilde{\beta}_{1}(y) u_{x}(x, y)+\tilde{h}(y) \text { on } \Gamma_{2} \\
& u(0,0)=\tilde{\gamma}(0,0)=\Gamma_{1} \cap \Gamma_{2} .
\end{aligned}
$$

The coefficients $\tilde{\alpha}_{k}, \tilde{\beta}_{k}, \tilde{g}, \tilde{h}$ and $\tilde{\gamma}$ are assumed to be complex valued.

By separating real and imaginary parts in equations (1.1) - (1.3) our results may be seen to apply to a system of real partial differential equations.

$$
\begin{aligned}
& \Delta u^{(k)}=\tilde{f}_{k}\left(x, y, u^{(1)}, u^{(2)}, u_{x}^{(1)}, u_{x}^{(2)}, u_{y}^{(1)}, u_{y}^{(2)}\right) \\
& (k=1,2)
\end{aligned}
$$

(*) This research was supported in part at the Institute for Fluid Dynamies and Applied Mathematies, University of Maryland, by the National Science Foundation under Grants GP.2067, GP-3937, and in part by the Air Force Office of Scientifie Research under Grant AFOSR 400-64, and at Georgetown University, Washington, D.C., by the National Science Foundation under Grant GP-1650 and GP-5023.

(i) Georgetown University, Washington, D.C.

(2) Indiana University, Bloomington, Indiana.

(3) University of Wisconsin-Milwaukee, Milwaukee, Wisconsin. 
satisfying four (real) generalized boundary conditions, namely, on $\Gamma_{1}$

$$
\begin{aligned}
& u_{x}^{(1)}=\alpha_{0}^{(1)} u^{(1)}-\alpha_{0}^{(2)} u^{(2)}+\alpha_{1}^{(1)} u_{y}^{(1)}-\alpha_{1}^{(2)} u_{y}^{(2)}+g^{(1)} \\
& u_{x}^{(2)}=\alpha_{0}^{(2)} u^{(1)}+\alpha_{0}^{(1)} u^{(2)}+\alpha_{1}^{(2)} u_{y}^{(1)}+\alpha_{1}^{(1)} u_{y}^{(2)}+g^{(2)} ; \text { on } \Gamma_{2} \\
& u_{y}^{(1)}=\beta_{0}^{(1)} u^{(1)}-\beta_{0}^{(2)} u^{(2)}+\beta_{1}^{(1)} u_{x}^{(1)}-\beta_{1}^{(2)} u_{y}^{(2)}-h^{(1)} \\
& u_{y}^{(2)}=\beta_{0}^{(2)} u^{(1)}+\beta_{0}^{(1)} u^{(2)}+\beta_{1}^{(2)} u_{x}^{(1)}+\beta_{1}^{(1)} u_{x}^{(2)}=h^{(2)} \\
& u^{(1)}(0,0)=\gamma^{(1)}, u^{(2)}(0,0)=\gamma^{(2)}
\end{aligned}
$$

Here, $\tilde{\alpha}_{k}(x) \equiv \alpha_{k}^{(1)}(x)+i \alpha_{k}^{(2)}(x), \tilde{\beta}_{k}(y) \equiv \beta_{k}^{(1)}(y)+i \beta_{k}^{(2)}(y)$,

$$
(k=0,1), u(x, y)=u^{(1)}(x, y)+i u^{(2)}(x, y) \text { ete. }
$$

The approach we shall use in this paper is similar to that used earlier by AzIz and GILBent [A.G. 1] to stady the linear elliptic partial differential equation,

$$
e(u) \equiv \Delta u+a(x, y) u_{x}+b(x, y) u_{y}+c(x, y) u=0,
$$

with boundary data $(1.4 \mathrm{a}$ b) and (1.5 a.b.c).

Our results in the case of equations (1.1) and (1.6) may be considered as an extension and generalization of the results of BERGMAN [B. 1] [B. 2], [B.S. 1] and of VekUA [V. 1], [V. 2], [H. 1] who have considered equation (1.6) for certain special boundary conditions. Both Bergman and VeKua introduce the complex variables $z=x+i y, z^{*}=x-i y$ and formally trans. form $e(u)=0$ into an associated hyperbolic equation,

$$
E(U)=U_{z z^{*}}+A\left(z, z^{*}\right) U_{z^{*}}+B\left(z, z^{*}\right) U_{z^{*}}+C\left(z, z^{*}\right) U=0 .
$$

VEKUA represents the solution of (1.7) in terms of its RIEMANN function for $\left(z, z^{*}\right)$ in a bicylinder $(S, \bar{S}) \subset C^{(2)}$ where the coefficients of (1.7) are assumed to be holomorphic functions of the two complex variables $z$ and $z^{*}$. (We use $\bar{S}$ here and afterwards to stand for the set $\left\{z^{*} \mid z^{*}=\bar{z}, z \in S\right\}$. Bergman, on the other hand, introduces an associated differential equation

$$
\left(1-t^{2}\right) \tilde{\varepsilon}_{z^{*} t}-\frac{1}{t} \tilde{\varepsilon}_{z^{*}}+2 t z\left[\tilde{\varepsilon}_{z_{z^{*}}}+D \tilde{\varepsilon}_{z^{*}}+F \tilde{\varepsilon}\right]=0
$$

where $D=n^{\prime}(z)-\int_{0}^{z^{*}} A_{z} d z^{*}+B, F=-A_{z}-A B+C$, and $n(z)$ is an arbitrary 
analytic function of $z \in S$. He is then able to represent solutions of (1.7) in the form [B. 1],

$$
U\left(z, z^{*}\right)=\int_{-1}^{+1} \varepsilon\left(z, z^{*}, t\right) f\left(\frac{z}{2}\left[1-t^{2}\right]\right) \frac{d t}{\sqrt{1-t^{2}}},
$$

where $\varepsilon\left(z, z^{*}, t\right)=\exp \left[-\int_{0}^{z^{*}} A d z^{*}+n(z)\right] \tilde{\varepsilon}\left(z, z^{*}, t\right)$.

BERGMAN [B. 1] was the first to show that if the coefficients of (1.6) are real analytic in a common domain $S$ of the $z$-plane, and if furthermore they have a holomorphic continuation to a bicylinder $(S, \bar{S})$ in the variable $\left(z, z^{*}\right)$, then any solution of $(1.6)$ of class $C^{2}$ has a holomorphic continuation to $(S, \bar{S})$ in the variables $\left(z, z^{*}\right)$. Later a different proof of this result was given by VEKUA [B. 1] [V. 1] [H. 1]. By introducing a suitable BANACH space we are able to generalize results of BERGMAN and VEKUA to the case of second order, non-linear equations (1.1), with boundary data of the form (1.2), (1.3).

\section{2. - The Associated Hyperbolic Problem.}

Consider the following elliptic boundary value problem:

$$
\Delta u=\tilde{f}\left(x, y, u, u_{x}, u_{y}\right), \text { with }
$$

$$
u_{x}(x, y)=\tilde{\alpha}_{0}(x) u(x, y)+\tilde{\alpha}_{1}(x) u_{y}(x, y)+\tilde{g}(x)
$$

on the curve $\Gamma_{1}=\left\{(x, y) \mid y=f_{2}(x), o \leq x \leq a\right\}$

$$
u_{y}(x, y)=\tilde{\beta}_{0}(y) u(x, y)+\tilde{\beta}_{1}(y) u_{x}(x, y)+\tilde{k}(y)
$$

on the curve $\left.\Gamma_{2}=\{x, y) \mid x=f_{2}(y), o \leq y \leq b\right\}$

$$
\text { and } u(0,0)=\tilde{\gamma}
$$

Now replace $x$ and $y$ by

$$
x=\frac{z+z^{*}}{2}, y=\frac{z-z^{*}}{2 i},
$$

with $z, z^{*}$ new independent complex variables. 
A simple chain rule computation shows that, formally,

$$
\begin{aligned}
& u_{x}=U_{z}+U_{z^{*}}, u_{\nu}=i\left(U_{z}-U_{z^{*}}\right) \\
& \Delta u=4 U_{z *^{*}} \quad \text { where } \quad u(x, y) \equiv U\left(z, z^{*}\right),
\end{aligned}
$$

and equations (2.1) becomes

$$
U_{z^{*}}=f\left(z, z^{*}, U, U_{z}, U_{z^{*}}\right)
$$

We now make assumptions concerning the functions appearing in the equation and boundary data. First we shall assume that $f$ as a function of its first two arguments is analytic and bounded in some bycylinder $(S, \bar{S}$ ) (where $S$ is a bounded domain) and as a function of its last three arguments is holo. morphic in a neighborhood of the origin, to be specified more precisely below. Second, it is assumed that the sets $\Gamma_{1}$ and $\Gamma_{2}$ under the transformation (2.3) can be represented, respectively, as

$$
\begin{aligned}
& \Gamma_{1} \equiv\left\{\left(z, z^{*}\right) \mid z^{*}=F_{1}(z)\right\}, \\
& \Gamma_{2} \equiv\left\{\left(z, z^{*}\right) \mid z=F_{2}\left(z^{*}\right)\right\},
\end{aligned}
$$

where $\Gamma_{1}$ and $\Gamma_{2}$ are contained in $(S, \bar{S})$ for some holomorphic functions $F_{1}$ and $F_{2}$ with the property that $F_{1}(0)=F_{2}(0)=0$. Finally we assume that the coefficients of the boundary data $\tilde{\alpha}_{i}, \tilde{\beta}_{i}(i=0,1)$ and $\tilde{g}, \tilde{h}$ have holomorphic continuations to $(S, \bar{S})$, that $\tilde{\alpha}_{i} \tilde{g}, f_{1}$ are holomorphic in

$$
S_{1} \equiv\left\{\xi \mid \xi=\frac{z+z^{*}}{2}, z \in S, z * \in \bar{S}, z \text { and } z^{*} \text { independent variables }\right\} \text {, }
$$

and that $\tilde{\beta}_{i}, \tilde{h}, f_{2}$ are holomorphie in

$$
S_{2} \equiv\left\{\xi \mid \xi=\frac{z-z^{*}}{2 i}, z \in S, z^{*} \in \bar{S}, z \text { and } z^{*} \text { independent variables }\right\}
$$

Such a bicylinder $(S, \vec{S})$ where these three assumptions hold will be called a fundamental bicylinder for equation (2.5) and curves (2.6).

Thus in place of the problem $(2.1-(2.2 \mathrm{a} \cdot \mathrm{c})$ we have

$$
\begin{aligned}
& U_{z^{*}}=f\left(z, z^{*}, U, U_{z}, U_{z^{*}}\right) \\
& U_{z}\left(z, z^{*}\right) \equiv \alpha_{0}\left(z, z^{*}\right) U\left(z, z^{*}\right)+\alpha_{1}\left(z, z^{*}\right) U_{z^{*}}\left(z, z^{*}\right) \\
& +g\left(z, z^{*}\right) \text { on } z^{*}=F_{1}(z)
\end{aligned}
$$




$$
\begin{aligned}
U_{z^{*}}\left(z, z^{*}\right) \equiv \beta_{0}\left(z, z^{*}\right) U\left(z, z^{*}\right) & +\beta_{1}\left(z, z^{*}\right) U_{z}\left(z, z^{*}\right) \\
& +h\left(z, z^{*}\right) \text { on } z=F_{2}\left(z^{*}\right)
\end{aligned}
$$

$$
\text { and } U(0,0)=\gamma(0,0) \in(S, \bar{S}) \text {. }
$$

where for example $\alpha_{k}\left(z, z^{*}\right)$ etc., is obtained under the transformation (2.3); see formulas $(5.5 a-c)$ and $(5.6 a-c)$ of this paper.

\section{3. - Preliminary Lemmas.}

Consider the set of functions $\mathfrak{Q} \equiv\{s \mid s \in H B(S, \bar{S})\}$ where $H B(S, \bar{S})$ denotes the class of functions of two complex variables $\left(z, z^{*}\right)$ which are holomorphic and bounded in $(S, \bar{S})$. If one introduces a norm in $\mathfrak{A}$ by

$$
\|s\|_{\lambda}=\sup \left\{e^{-\lambda\left(|z|+\left|z^{*}\right|\right)} \mid s\left(z, z^{*} \mid\right\}\right.
$$

where $\left(z, z^{*}\right) \in(S, \bar{S})$ and $\lambda>0$ is fixed then is is easy to verify that $\mathfrak{A}$ is a normed linear vector space. Moreover, modifying the proof given by HiLuE, [H. 2], pgs. 192-193 for the completeness of $H B(S)$ (the set of functions of one complex variable which are holomorphic and bounded in the domain $S$ ) by replacing the single integrals by double CAUCHY integrals (see FuKs [F. 1], pg. 32) one concludes that $\mathfrak{A}$ is also a complete space. The details of these calculations may be readily supplied by the reader. Hence we conclude $\mathfrak{A}$ is a BANAOH space, by definition.

Suppose now $U$ is a solntion of $(2.8)$ and define a function $s\left(z, z^{*}\right)(\varepsilon \mathfrak{d})$ by

$$
U_{z z^{*}}=s
$$

We then have

$$
\begin{aligned}
& U\left(z, z^{*}\right)=\int_{0}^{z} \int_{0}^{\hat{z}^{*}} s\left(\xi, \xi^{*}\right) d \xi^{*} d \xi+ \\
& +\int_{0}^{z} \varphi(\xi) d \xi+\int_{0}^{z^{*}} \psi\left(\xi^{*}\right) d \xi^{*}+U(0,0), \\
& U_{z}\left(z, z^{*}\right)=\int_{0}^{z^{*}} s\left(z, \xi^{*}\right) d \xi *+\varphi(z), \\
& U_{z^{*}}\left(z, z^{*}\right)=\int_{0}^{z} s\left(\xi, z^{*}\right) d \xi+\psi\left(z^{*}\right),
\end{aligned}
$$


where

$$
\varphi(z)=U_{z}(z, 0), \biguplus\left(z^{*}\right)=U_{z^{*}}\left(0, z^{*}\right)
$$

We note that $s\left(z, z^{*}\right)$ must satisfy the equation

$$
\begin{aligned}
s\left(z, z^{*}\right)= & f\left(z, z^{*}, \int_{0}^{z} \int_{0}^{\xi^{*}} s\left(\xi, \xi^{*}\right) d \xi * d \xi+\int_{0}^{z} \varphi(\xi) d \xi+\right. \\
+ & \int_{0}^{z^{*}} \psi\left(\xi^{*}\right) d \xi^{*}+U(0,0), \int_{0}^{z^{*}} s\left(z, \xi^{*}\right) d \xi^{*}+\varphi(z), \\
& \left.\int_{0}^{\xi} s\left(\xi, z^{*}\right) d \xi+\psi\left(z^{*}\right)\right) .
\end{aligned}
$$

Conversely, if $s$ satisfies (3.7) then a solution of (2.7) is given by (3.3). We also find that the boundary.conditions $(2.8 \mathrm{a} \cdot \mathrm{c})$ are equivalent to

$$
\begin{aligned}
\varphi(z) & =\alpha_{0}(z)\left\{\int_{0}^{z} \varphi(\xi) d \xi+\int_{0}^{F_{1}(z)} \psi\left(\xi^{*}\right) d \xi^{*}+\right. \\
& \left.+\int_{0}^{z} \int_{0}^{F_{1}(z)} s\left(\xi, \xi^{*}\right) d \xi^{*} d \xi+\gamma\right\}+\alpha_{1}(z)\left\{\psi\left(F_{1}(z)\right)+\right. \\
& \left.+\int_{0}^{z} s\left(\xi, F_{1}(z)\right) d \xi\right\}-\int_{0}^{F_{1}(z)} s\left(z, \xi^{*}\right) d \xi *+g(z)
\end{aligned}
$$

and

$$
\begin{aligned}
\psi(z) & =\beta_{0}\left(z^{*}\right)\left\{\int_{0}^{z^{*}} \psi\left(\xi^{*}\right) d \xi^{*}+\int_{0}^{F_{2}\left(z^{*}\right)} \varphi(\xi) d \xi+\right. \\
& \left.+\int_{0}^{F_{2}\left(z^{*}\right)} \int_{0}^{z^{*}} s\left(\xi, \xi^{*}\right) d \xi^{*} d \xi+\gamma\right\}+\beta_{1}\left(z^{*}\right)\left\{\varphi\left(F_{2}\left(z^{*}\right)\right)+\right. \\
& \left.+\int_{0}^{z^{*}} s\left(F_{2}\left(z^{*}\right), \xi^{*}\right) d \xi^{*}\right\}-\int_{0}^{F_{2}\left(z^{*}\right)} s\left(\xi, z^{*}\right) d \xi+h\left(z^{*}\right) .
\end{aligned}
$$

We have thus demonstrated the following lemma. 
Aziz - Gilbert - Howard: A second order, non-linear elliptic, ete. 331

LEMMA 1. - If $U$ is a solution of equation (2.7) then $s$ as given by (3.2) satisfies (3.7), and conversely. Moreover the boundary condition (2.8a-c) are equivalent to (3.8a-b) under the transformation (3.2).

Next consider the BANAOH spaces $\mathfrak{G}_{1}$ and $\mathfrak{G}_{2}$ of functions holomorphic and bounded in $S$ and $\bar{S}$, with norms given by, respectively,

and

$$
\|\varphi\|_{\lambda}=\sup \left\{e^{-\lambda|z|}|\varphi(z)|\right\} \quad \text { where } z \in S, \lambda>0 \text {, }
$$

$$
\|\psi\|_{\lambda}=\sup \left\{e^{-\lambda\left|z^{*}\right|}\left|\Psi\left(z^{*}\right)\right|\right\} \text { where } z^{*} \in \bar{S}, \lambda>0 \text {. }
$$

For convenience we introduce the following notation

$$
F=(\varphi, \psi), F_{0}=\left(g(z)+\alpha_{0}(z) \gamma, h\left(z^{*}\right)+\beta_{0}\left(z^{*}\right) \gamma\right)
$$

$$
H F=\left(\alpha_{1}(z) \psi\left(F_{1}(z)\right), \beta_{1}\left(z^{*}\right) \varphi\left(F_{2}\left(z^{*}\right)\right)\right.
$$

$$
\begin{array}{r}
\mathcal{H} F=\left(\alpha_{0}(z)\left\{\int_{0}^{z} \varphi(\xi) d \xi+\int_{0}^{F_{1}(z)} \psi\left(\xi^{*}\right) d \xi^{*}\right\},\right. \\
\left.\beta_{0}\left(z^{*}\right)\left\{\int_{0}^{z^{*}} \psi\left(\xi^{*}\right) d \xi^{*}+\int_{0}^{F_{2}\left(z^{*}\right)} \varphi(\xi) d \xi\right\}\right)
\end{array}
$$

$$
\begin{aligned}
\Omega s= & \left(\Omega_{1} s, \Omega_{2} s\right)= \\
= & \left(\alpha_{0}(z) \int_{0}^{z} \int_{0}^{F_{1}(z)} s\left(\xi, \xi^{*}\right) d \xi * d \xi+\alpha_{1}(z) \int_{0}^{z} s\left(\xi, F_{1}(z)\right) d \xi-\int_{0}^{F_{1}(z)} s\left(z, \xi^{*}\right) d \xi^{*}\right. \\
& \beta_{0}\left(z^{*}\right) \int_{0}^{F_{2}\left(z^{*}\right)} \int_{0}^{z^{*}} s\left(\xi, \xi^{*}\right) d \xi * d \xi+\beta_{1}\left(z^{*}\right) \int_{0}^{z^{*}} s\left(F_{2}\left(z^{*}\right), \xi^{*}\right) d \xi^{*}- \\
& \left.-\int_{0}^{F_{2}\left(z^{*}\right)} s\left(\xi, z^{*}\right) d \xi\right) .
\end{aligned}
$$

We may then write system (3.8) symbolically in operator notation as

$$
(I-H-\mathscr{H}) F=F_{0}+\Omega s
$$

and if the inverse operator $\mathfrak{B}=\left(I-H-\mathfrak{H}^{-1}\right.$ exists then we shall be able to express the functions $\varphi$ and $\psi$ as operators on the function $s$. Note that 
in the present context we are dealing with couples $(\varphi, \psi)$, etc., where $\varphi \in \mathfrak{A}_{1}$, $\psi \in \mathfrak{Q}_{2}$ and that this set of couples may be itself regarded as a BANACH space if one uses as a norm $\|(\varphi, \psi)\|=\max \{\|\varphi\|,\|\psi\|\}$. Let us denote this BANACH space by $C(S, \bar{S})$. We note that the various operators we are considering are from $O$ into $C$ if $\left(F_{1}(z), F_{2}\left(z^{*}\right) \subset(S, \bar{S})\right.$.

We turn our attention now to showing that $\mathscr{B}$ exists. It is sufficient to show that the equation

$$
(I-H-\mathfrak{H}) F=G
$$

has a unique solution for every $G \in C$. Now if $(I-H)^{-1}$ exists the last equation is equivalent to

$$
\left(I-(I-H)^{-1} \mathcal{H}\right) F=(I-H)^{-1} G
$$

We thus consider the problem of showing that $(I-H)^{-1}$ exists. Noting the definition of $H$ we have

$$
H^{2} F=\left(\gamma_{1}(z) \varphi\left(\Lambda_{1}(z)\right), \gamma_{2}\left(z^{*}\right) \psi\left(\Lambda_{2}\left(z^{*}\right)\right)\right)
$$

for $F=(\varphi, \psi)$, where

$$
\begin{aligned}
& \gamma_{1}(z)=\alpha_{1}(z) \beta_{1}\left(F_{1}(z)\right) \quad \Lambda_{1}(z)=F_{2}\left(F_{1}(z)\right) \\
& \gamma_{2}\left(z^{*}\right)=\beta_{1}\left(z^{*}\right) \alpha_{1}\left(F_{2}\left(z^{*}\right)\right) \quad \text { and } \quad \Lambda_{2}\left(z^{*}\right)=F_{1}\left(F_{2}\left(z^{*}\right)\right)
\end{aligned}
$$

Thus if $H^{2 n} F^{\prime}=(\tilde{\varphi}, \tilde{\psi})$ we get

$$
\begin{aligned}
& \tilde{\varphi}(z)=\gamma_{1}^{(n)}(z) \varphi\left(\Lambda_{1}^{(n)}(z)\right) \\
& \tilde{\Psi}\left(z^{*}\right)=\gamma_{2}^{(n)}\left(z^{*}\right) \psi\left(\Lambda_{2}^{(n)}\left(z^{*}\right)\right)
\end{aligned}
$$

where

and

$$
\begin{aligned}
& \Lambda_{1}^{(n)}(z)=\Lambda_{1}\left(\Lambda_{1}^{(n-1)}(z)\right) \quad n=1,2, \ldots \quad \Lambda_{1}^{(o)}(z)=z \\
& \gamma_{1}^{(n)}(z)=\gamma_{1}(z) \gamma_{1}\left(\Lambda_{1}(z)\right) \ldots \gamma_{1}\left(\Lambda_{1}^{(n-1)}(z)\right)
\end{aligned}
$$

$$
\Lambda_{2}^{(n)}\left(z^{*}\right) \quad \text { and } \quad \gamma_{2}^{(n)}\left(z^{*}\right)
$$

are defined similarly. We see that

$$
\begin{aligned}
& \|\tilde{\varphi}\|_{\lambda} \leq\left\|\gamma_{i}^{(n)}\right\|_{0}\|\varphi\|_{\lambda} \\
& \|\tilde{\Psi}\|_{\lambda} \leq\left\|\gamma_{1}^{(n)}\right\|_{0}\|\psi\|_{\lambda}
\end{aligned}
$$


and hence

$$
\left\|H^{2 n}\right\|_{\lambda} \leq \max \left\{\left\|\gamma_{1}^{(n)}\right\|_{0}, \|\left.\gamma_{2}^{(n)}\right|_{0}\right\}, \lambda \geq 0
$$

We assume that $S$ contains the set $\Delta \rho \equiv\{z|| z \mid \leq \rho\}$ and that $\bar{S}$ contains the set $\Delta \rho^{*} \equiv\left\{z^{*}|| z^{*} \mid \leq \rho\right\}$. We also assume that $F_{1}$ maps $\Delta \rho$ into $\Delta \rho^{*}$ and that $F_{2}$ maps $\Delta \rho^{*}$ into $J_{\rho}$; we have assumed $F_{1}(0)=0, F_{2}(0)=0$. The functions $\Lambda_{1}(z) \equiv F_{2}\left(F_{1}(z)\right)$ and $\Lambda_{2}(z) \equiv F_{1}\left(F_{2}\left(z^{*}\right)\right)$ have similar properties. Applying the SoHwarz lemma for functions of one complex variable we have $\left|F_{1}(z)\right| \leq$ $\leq|z|,\left|F_{2}\left(z^{*}\right)\right| \leq\left|z^{*}\right|$ with equality occurring only if $F_{1}(z)=e^{i x_{z}}$, or $F_{2}\left(z^{*}\right)=e^{i z^{*}}$, $\alpha$ and $\beta$ real. Returning to the definition of $z^{*}=F_{1}(z)$, for example, $\left(z+z^{*}\right) / 2=f_{1}\left(\left(z-z^{*}\right) / 2 i\right)$ we realize it is impossible for $F_{1}(z)$ to be of the form $F_{1}(z) \equiv C_{1} z$ unless $y=f_{1}(x)$ is a straight line $y=A x$. In this case $F_{1}(z)=(i+A) /(i-A) z=e^{i x} z$ since $A$ is taken to be real. A similar remark holds for $F_{2}\left(z^{*}\right)$. Consequently, unless $f_{1}(x) \equiv A x$ and $f_{2}(y) \equiv B y \quad(A, B$ real) either $F_{1}$ satisfies the condition $\left|F_{1}(z)\right|<z$ or $F_{2}\left(z^{*}\right)$ satisfies the condition $\left|F_{2}\left(z^{*}\right)\right|<z^{*}$. We conclude

$$
\left|\Lambda_{1}(z)\right|<|z|,\left|\Lambda_{2}\left(z^{*}\right)\right|<\left|z^{*}\right| \text {. }
$$

We assume that not both of the curves $y=f_{1}(x), x=f_{2}(y)$ are straight lines through the origin. We note that, $\left|\Lambda_{1}(z)\right|<|z|$ implies $\left|\Lambda_{1}(z)\right| \leq \varepsilon|z|, 0<\varepsilon<1$, so $\left|\Lambda_{1}^{(2)}(z)\right|=\left|\Lambda_{1}\left(\Lambda_{1}^{(1)}(z)\right)\right| \leq \varepsilon\left|\Lambda_{1}^{(1)}(z)\right| \leq \varepsilon^{2}|z|$ and in general

$$
\left|\Lambda_{1}^{(n)}(z)\right| \leq \varepsilon^{n}|z|, \text { with a similar formula for }\left|\Lambda_{2}^{(n)}\left(z^{*}\right)\right|
$$

Hence $\left|\Lambda_{1}^{(n)}\right|$ and $\left|\Lambda_{2}^{(n)}\right|$ converge uniformly to 0 on $\Delta_{p}$ and $\Delta_{0}^{*}$.

Next let us assume that $\left|\gamma_{1}(0)\right|=\left|\gamma_{2}(0)\right|=\left|\alpha_{1}(0) \beta_{1}(0)\right|<1$ and observe that since $\gamma_{2}$ and $\gamma_{2}$ are continuous there exists a positive number $\varepsilon<1$, and a $\delta>0$, sufficiently small, such that

$$
\left|\gamma_{1}(z)\right|<\varepsilon^{2},\left|\gamma_{2}\left(z^{*}\right)\right|<\varepsilon^{2}
$$

when $|z| \leq \delta,\left|z^{*}\right| \leq \delta$. Moreover, for $n \geq n_{0}$, say, with $n_{0}$ sufficiently large, we have

$$
\left|\Lambda_{1}^{(n)}(z)\right| \leq \delta,\left|\Lambda_{2}^{(n)}\left(z^{*}\right)\right| \leq \delta
$$

where $\left(z, z^{*}\right) \subset\left(\Delta_{\rho}, \Delta_{\rho}^{*}\right)$. Now for $n \geq n_{0}$ we have

$$
\begin{aligned}
& \left|\gamma_{1}^{(n)}(z)\right| \leq\left|\gamma_{1}(z)\right|\left(\varepsilon^{2}\right)^{n-n_{0}}, \\
& \left|\gamma_{2}^{(n)}\left(z^{*}\right)\right| \leq\left|\gamma_{2}\left(z^{*}\right)\right|\left(\varepsilon^{2}\right)^{n-n_{0}}
\end{aligned}
$$


by use of (3.18) and (3.24). But from (3.17) one has

so

$$
\begin{aligned}
& \left|\gamma_{1}(z)\right| \leq\left\|\alpha_{1}\right\|_{0}\left\|\beta_{1}\right\|_{0} \\
& \left|\gamma_{2}\left(z^{*}\right)\right| \leq\left\|\alpha_{1}\right\|_{0}\left\|\beta_{1}\right\|_{0}
\end{aligned}
$$

$$
\left\|H^{2 n}\right\|_{\lambda} \leq\left\{\left\|\alpha_{1}\right\|_{0}\left\|\beta_{1}\right\|_{0} \varepsilon^{-2 n_{0}}\right\}\left(\varepsilon^{2}\right)^{n}, n \geq n_{0}
$$

Moreover for $n<n_{0}$,

$$
\begin{aligned}
\left\|H^{2 n}\right\|_{\lambda} & \leq \max \left\{\left\|\gamma_{1}^{(n)}\right\|_{0},\left\|\gamma_{2}^{(n)}\right\|_{0}\right\} \\
& \leq \max \left\{\left\|\gamma_{1}^{(n)}\right\|_{0},\left\|\gamma_{2}^{(n)}\right\|_{0}\right\} \times \\
& \times\left(\varepsilon^{2}\right)^{-n}\left(\varepsilon^{2}\right)^{n}
\end{aligned}
$$

since $0<\varepsilon^{2}<1$ and $n<n_{0}$.

Hence if $M=\max \left(M_{1}, M_{2}\right\}$, where

we have

$$
\begin{aligned}
& M_{1}=\left\|\alpha_{1}\right\|_{0}\left\|\beta_{1}\right\|_{0} \varepsilon^{-2 n_{0}} \\
& M_{2}=\max _{n \leq n_{0}}\left\{\frac{\left\|\gamma_{1}^{(n)}\right\|_{0}}{\varepsilon^{2 n}}, \frac{\left\|\gamma_{2}^{(n)}\right\|_{0}}{\varepsilon^{2 n}}\right\}
\end{aligned}
$$

$$
\begin{aligned}
& \left\|H^{2 n}\right\|_{\lambda} \leq M \varepsilon^{2 n} \text {. But } \\
& \left\|H^{2 n+1}\right\|_{\lambda}=\left\|H^{2 n} H^{1}\right\|_{\lambda} \leq M \varepsilon^{2 n+1} \max \left\{\frac{\left\|\alpha_{1}\right\|_{0}}{\varepsilon}, \frac{\left\|\beta_{1}\right\|_{0}}{\varepsilon}\right\} .
\end{aligned}
$$

Setting $N=\max \left\{M, \frac{M\left\|\alpha_{1}\right\|}{\varepsilon}, \frac{M\left\|\beta_{1}\right\|}{\varepsilon}\right\}$ we have

$$
\left\|H^{n}\right\| \leq N \varepsilon^{n} \quad n=1,2, \ldots
$$

and the series $I+H+H^{2}+\ldots$ therefore converges. Thas $(I-H)^{-1}$ exists, and from the series expansion for $(I-H)^{-1}$ we conclude there exists a positive constant $k$ such that $\left\|(I-H)^{-1}\right\|_{\lambda} \leq k$.

We summarize this analysis in the following lemma.

Lemma 2. - If

1. $S \supset \Delta \rho$ and $\bar{S} \supset \Delta * \rho$,

2. $F_{1}$ maps $\Delta \rho$ into $\Delta * \rho, F_{2}$ maps $\Delta * \rho$ into $\Delta \rho$, and $F_{1}$ and $F_{2}$ leave the origin fixed, 
3. $\left|\alpha_{1}(0) \beta_{1}(0)\right|<1$,

4. either $y=f_{1}(x)$ or $x=f_{2}(y)$ is not a straight line (with real coefficients) through the origin, then the operator $\left(I-H^{-1}\right.$ exists in the Banach spuce $C\left(\Delta \rho, \Delta^{*} \rho\right)$ and $\left\|(I-H)^{-1}\right\|_{\lambda} \leq k$ for some positive constant $k$, and any $\lambda>0$.

Returning now to the problem of solving equation (3.14), or (3.15) we set

$$
A \equiv(I-H)^{-1} \mathfrak{H}
$$

noting that $A$ exists since if $x \in C(S, S)$ and $y=\mathscr{H} x$ then $(I-H)^{-1} y$ is well-defined. Then one has $\|A\|_{\lambda} \leq\left\|(T-H)^{-1}\right\|_{\lambda}\|\mathfrak{H}\|_{\lambda}$. If we let $\tilde{F}=\mathfrak{H} F=(\tilde{\varphi}, \tilde{\varphi})$, then from the definition of $\mathcal{H}$, we have

$$
\begin{aligned}
|\varphi(z)| & =\left|\alpha_{0}(z)\left\{\int_{0}^{z} \varphi(\xi) d \xi+\int_{0}^{F_{1}(z)} \psi\left(\xi^{*}\right) d \xi^{*}\right\}\right| \\
& \leq\left\|\alpha_{0}\right\|_{0}\left\{\left|\int_{0}^{z} \varphi(\xi) d \xi\right|+\left|\int_{0}^{F_{1}(z)} \psi\left(\xi^{*}\right) d \xi^{*}\right|\right\} \\
& \leq\left\|\alpha_{0}\right\|_{0}\left\{\int_{0}^{|z|}\|\varphi\|_{\lambda} e^{\lambda|\xi|}+\int_{0}^{|z|}\|\psi\|_{\lambda} e^{\lambda\left|\xi^{*}\right|}\left|d \xi^{*}\right|\right\} \\
& \leq 2\left\|\alpha_{0}\right\|_{0} \frac{e^{\lambda|z|}}{\lambda}\|F\|_{\lambda,}
\end{aligned}
$$

where we have used the facts that $\left|F_{1}(z)\right|<|z|$, and that $\varphi$ and $\psi$ are regular in the circles $\Delta p$ and $\Delta * \rho$ so that curvilinear paths of integration may be replaced by straight line paths of integration.

Similarly for $\left|\tilde{\psi}\left(z^{*}\right)\right|$ we have

$$
\left|\tilde{\psi}\left(z^{*}\right)\right| \leq 2\left\|\beta_{0}\right\|_{0} \frac{e^{\lambda|z|}}{\lambda}\|F\|_{\lambda}
$$

so if $\rho$ is chosen sufficiently small, i.e. $\rho=\rho(\lambda)$, then

$$
\|\mathscr{H}\|_{\lambda} \leq \frac{M}{\lambda}
$$

for some positive constant $M$, and $\lambda>0$. Combining this result with $\left\|(I-H)^{-1}\right\|_{\lambda} \leq k$ we have, for sufficiently large $\lambda$,

$$
\|A\| \leq k M / \lambda<\frac{1}{2}
$$


so the operator $(I-A)^{-1}=I+A+A^{2}+\ldots$ exists and its norm satisfies $\left\|(I-A)^{-1}\right\|_{\lambda} \leq 2$ for $\lambda$ sufficiently large. Hence the operator

$$
\mathscr{B}=(1-H-\mathfrak{H})^{-1}=(I-A)^{-1}(I-H)^{-1}
$$

exists for $\lambda$ sufficiently large and we have

$$
\|\mathfrak{B}\|_{\lambda} \leq 2 \cdot k \text {. }
$$

Thus we see that the solution of (3.13) is given by

$$
\begin{aligned}
F & =\mathscr{B} F_{0}+\mathscr{B} \Omega s \\
& \equiv\left(\varphi_{1}: \psi_{1}\right)+\left(\mathfrak{B}_{1} \Omega_{1} s, \mathfrak{B}_{2} \Omega_{2} s\right) .
\end{aligned}
$$

We have the following lemma.

Lemma 3. - If the hypotheses of Lemma 2 hold, then equation (3.13) has a solution $F$ in the Banach space $C\left(\Delta \rho, \Delta^{*} \rho\right)$, given by (3.37).

\section{4. - The Fundamental Existenee Theorem.}

With these preliminarly considerations taken care of, we turn to the main problem of establishing the existence of a unique solution to the problem given by $(2.1) \cdot(2.2 \mathrm{a}-\mathrm{c})$.

Let $T$ be the operator mapping the BANACH space $\mathfrak{A}_{\rho}$ into itself, where

$$
\mathfrak{Q}_{p} \equiv\left\{s \mid s \varepsilon H B\left(\Delta \rho, \Delta^{*} \rho\right)\right\}
$$

(see the introductory paragraph of Section III for notation), and

$$
T s\left(z, z^{*}\right)=f\left(z, z^{*}\right), B_{1} s\left(z, z^{*}, B_{2} s\left(z, z^{*}\right), B_{3} s\left(z, z^{*}\right)\right)
$$

where the $B_{i}$ are given by the right members of (3.3), (3.4), (3.5), respectively. Thus to prove existence and uniqueness of our original problem it is sufficient, using Lemmas 1 and 3 to show that the operator (4.1) has a unique fixed point in $\mathfrak{A}_{p}$ where the $\varphi$ and $\psi$ appearing in the definitions of the $B_{i}$ are given as the components of the right member of (3.37). (See [K.F. 1] for a discussion of the principle of contraction mappings and fixed points).

From the definition of the $B_{i}$ and (3.37) we have

$$
\begin{aligned}
B_{1} s & =D_{1} s+J_{1} \mathfrak{B}_{1} \Omega_{1} s+ \\
& +J_{2} \mathfrak{B}_{2} Q_{2} s+u_{1} \\
B_{2} s & =D_{2} s+\mathfrak{B}_{1} \Omega_{1} s+\varphi_{1} \\
B_{3} s & =D_{3} s+\mathscr{B}_{2} \Omega_{2} s+\psi_{1}
\end{aligned}
$$


where

$$
\begin{aligned}
& D_{1} s\left(z, z^{*}\right)=\int_{0}^{z} \int_{0}^{z^{*}} s\left(\xi, \xi^{*}\right) d \xi^{*} d \xi, \\
& D_{2} s\left(z, z^{*}\right)=\int_{0}^{z^{*}} s\left(z, \xi^{*}\right) d \xi^{*} \\
& D_{2} s\left(z, z^{*}\right)=\int_{0}^{z} s\left(\xi, z^{*}\right) d \xi \\
& J_{1} B_{1} \mathbf{Q}_{1} s\left(z, z^{*}\right)=\int_{0}^{z}\left(\mathscr{B}_{1} \mathbf{Q}_{1} s\right)\left(\xi, z^{*}\right) d \xi \\
& J_{2} B_{2} \mathbf{Q}_{2} s\left(z, z^{*}\right)=\int_{0}^{z^{*}} \mathscr{B}_{2} \mathrm{Q}_{2} s\left(z, \xi^{*}\right) d \xi^{*}
\end{aligned}
$$

and

$$
u_{1}\left(z, z^{*}\right)=\gamma+\int_{0}^{z} \varphi_{1}(\xi) d \xi+\int_{0}^{z^{*}} \psi_{1}\left(\xi^{*}\right) d \xi^{*}
$$

Bat it is an easy calculation to show that

$$
\begin{aligned}
\left|B_{1} s\right| & \leq\left\|D_{1}\right\| \lambda\|s\|_{\lambda}+\left\|J_{1} \mathscr{B}_{1} \Omega_{1}\right\|_{\lambda}\|s\|_{\lambda} \\
& +\left\|J_{2} \mathscr{B}_{2} \Omega_{2}\right\|_{\lambda}\|s\| \lambda+\left\|u_{1}\right\|_{0}
\end{aligned}
$$

where all the coefficients of $\|s\|_{\lambda}$ are $0(1 / \lambda)$ for $\lambda$ sufficiently large and $\rho=\rho(\lambda)$ taken sufficiently small (compare (3.31), for example). Hence if $\|s\|_{\lambda}$ is bounded and $\lambda$ is sufficiently large, $\left|B_{1} s\right|<k_{1}$ if $\left\|u_{1}\right\|_{0}<k_{1}$.

By completely analogous reasonings we have $\left|B_{2} s\right|<k_{2}$ and $\left|B_{3} s\right|<k_{3}$, for $\|s\|_{\lambda}$ bounded and $\lambda$ sufficiently large, where $\left\|\varphi_{1}\right\|_{0}<k_{2}$ and $\left\|\psi_{1}\right\|_{0}<k_{3}$.

We have assumed $f\left(z, z^{*}, u_{1}, u_{2}, u_{3}\right)$ is a holomorphic function of its first two arguments when $\left(z, z^{*}\right) \subset\left(\Delta \rho, \Delta^{*} \rho\right)$; assume further that as a function of its last three arguments it is holomorphic when

$$
\left|u_{i}\right| \leq k=\max \left(k_{1}, k_{2}, k_{3}\right), \quad i=1,2
$$

This last assumption is certainly true, no matter what value $k$ has, if $f$ is an entire function in the $u_{i}$. 
AzIz - GILbert - HowaRD: A second order, non-linear elliptic, etc.

Thus $f$ is holomorphic in a compact subset of $C^{(5)}$, and a LrPschirz condition, with respect to the last three arguments, holds there, so we have

$$
\begin{aligned}
& \left|f\left(z, z^{*}, u_{1}, u_{2}, u_{3}\right)\right| \leq\left|f\left(z, z^{*}, 0,0,0\right)\right| \\
& +1_{1}\left|u_{1}\right|+1_{2}\left|u_{2}\right|+1_{3}\left|u_{3}\right|, \quad 1_{i}=\text { const. }
\end{aligned}
$$

Combining this with the appraisals given above for $\left|B_{i} s\right|$ we conclude that, for $\|s\|_{\lambda}$ bounded and $\lambda$ sufficiently large,

$$
\begin{gathered}
\left|f\left(z, z^{*}, B_{1} s, B_{2} s, B_{3} s\right)\right| \leq \\
\left|f\left(z, z^{*}, 0,0,0\right)\right|+\left(1_{1}+1_{2}+1_{s}\right) \times k=R,
\end{gathered}
$$

say.

Thus if we assume

$$
\|s\|_{2} \leq R
$$

we see from (4.6) that $\|T s\|_{\lambda} \leq R$ for $\lambda$ sufficiently large. Hence $T$ is a mapping fom a closed subset of $\mathfrak{E}_{\rho}$ to $\mathfrak{E}_{\rho}$. Moreover, from the Lipschitz condition on $f$ we have

$$
\begin{aligned}
& \|T s-T \bar{s}\|_{\lambda} \leq C_{0}\left\{\left\|B_{1} s-B_{2} \bar{s}\right\|_{\lambda}\right. \\
& \left.\quad+\left\|B_{2} s-B_{2} \bar{s}\right\|_{\lambda}+\left\|B_{3} s-B_{3} \bar{s}\right\|_{\lambda}\right\}
\end{aligned}
$$

where $C_{0}$ is a positive constant. From arguments used several times before we have that

$$
\left\|B_{i} s-B_{i} \bar{s}\right\| \leq \frac{K_{i}}{\lambda}\|s-\bar{s}\|_{\lambda}
$$

where the $K_{i}$ are positive contants independent of $\lambda$, and $\lambda>0$. Hence for $\lambda$ sufficiently large we have

$$
\|T s-T \bar{s}\|_{\lambda}<\|s-\bar{s}\|_{\lambda}
$$

that is $T$ is a contraction mapping and the existence of a unique solution to the equation $T s=s$ in $\mathfrak{A}_{p}$ follows at once.

We have thus proved the following theorem, the desired existence theorem.

THEOREM 4.1. - If $(2.6 a \cdot b)$,

1. $(S, \bar{S})$ is a fundamental bicylinder for equation (2.5) and the curves

2. $S \supset \Delta \rho, \bar{S} \supset \Delta * \rho$, 
AzIZ - GILBERT - HowARD: A second order, non-linear elliptic, ete.

339

3. $F_{1}: \Delta \rho \rightarrow \Delta * p, \quad F_{2}: \Delta * p \rightarrow \Delta \rho$

4. $\left|\alpha_{1}(0) \beta_{1}(0)\right|<1$

5. $f\left(z, z^{*}, u_{1}, u_{2}, u_{3}\right)$ is an analitic function when $\left(z, z^{*}, u_{1}, u_{2}, u_{3}\right)$ $\varepsilon\left(\Delta \rho, \Delta \rho^{*} ; \Delta k, \Delta k, \Delta k\right)$, (cf. 4.5 for the definition of $k$ )

6. either $y=f_{1}(x) \neq A x$ or $x=f_{2}(y) \neq B y, A, B$, real constants, then the problem $(2.7)-(2.8 \mathrm{a} \cdot \mathrm{c})$ has a unique solution in $\left(\Delta \rho, \Delta^{*} \rho\right)$.

\section{Concluding Remarks and Interpretation of Results.}

As remarked earlier the elliptic boundary value problem

$$
\Delta u=\tilde{f}\left(x, y, u, u_{x}, u_{y}\right)
$$

with

$$
u_{x}(x, y)=\tilde{\alpha}_{0}(x) u(x, y)+\tilde{\alpha}_{1}(x) u_{y}(x, y)+\tilde{g}(x)
$$

on the curve $y=f_{1}(x)$

$$
u_{y}(x, y)=\tilde{\beta}_{0}(y) u(x, y)+\tilde{\beta}_{1}(y) u_{x}(x, y)+\tilde{h}(y)
$$

on the curve $x=f_{2}(y)$

$$
u\left(x_{0}, y_{0}\right)=\tilde{\gamma}
$$

becomes the following «hyperbolic problem»

$$
\begin{aligned}
U_{z}\left(z, z^{*}\right)=\alpha_{0}\left(z, z^{*}\right) U\left(z, z^{*}\right) & +\alpha_{1}\left(z, z^{*}\right) U_{z^{*}}\left(z, z^{*}\right) \\
& +g\left(z, z^{*}\right) \text { on } z^{*}=F_{1}(z) \\
U_{z^{*}}\left(z, z^{*}\right)=\beta_{0}\left(z, z^{*}\right) U\left(z, z^{*}\right) & +\beta_{1}\left(z, z^{*}\right) U_{z}\left(z, z^{*}\right) \\
& +h\left(z, z^{*}\right) \text { on } z=F_{2}\left(z^{*}\right)
\end{aligned}
$$

and

$$
U(0,0)=\gamma
$$


where

$$
\begin{aligned}
& \alpha_{0}(z)=\tilde{\alpha}_{0}\left(\frac{z+F_{1}(z)}{2}\right) /\left[1-i \tilde{\alpha}_{1}\left(\frac{z+F_{1}(z)}{2}\right)\right] \\
& \alpha_{1}(z)=\left[i \tilde{\alpha}_{1}\left(\frac{z+F_{1}(z)}{2}\right)+1\right] /\left[i \tilde{\alpha}_{1}\left(\frac{z+F_{1}(z)}{2}\right)-1\right] \\
& g(z)=\tilde{g}\left(\frac{z+F_{1}(z)}{2}\right) /\left[i \tilde{\alpha}_{1}\left(\frac{z+F_{1}(z)}{2}\right)-1\right]
\end{aligned}
$$

and where

$$
\begin{aligned}
& \beta_{0}\left(z^{*}\right)=-\tilde{\beta}_{0}\left(\frac{z^{*}-F_{2}\left(z^{*}\right)}{2}\right) /\left[\tilde{\beta}_{1}\left(\frac{z^{*}-F_{2}\left(z^{*}\right)}{2}\right)+i\right], \\
& \beta_{1}\left(z^{*}\right)=\left[i-\beta_{1}\left(\frac{z^{*}-F_{2}\left(z^{*}\right)}{2 i}\right)\right] /\left[\tilde{\beta}_{1}\left(\frac{z^{*}-F_{2}\left(z^{*}\right)}{2 i}\right)+i\right], \\
& h\left(z^{*}\right)=-\tilde{h}\left(\frac{z^{*}-F_{2}\left(z^{*}\right)}{2 i}\right) /\left[\tilde{\beta}_{1}\left(\frac{z^{*}-F_{2}\left(z^{*}\right)}{2 i}\right)+i\right] .
\end{aligned}
$$

The condition (4) of Theorem 4.1 becomes, in terms of $\tilde{\alpha}_{1}(0)$ and $\tilde{\beta}_{1}(0)$, the following

$$
\left|\frac{\tilde{\alpha}_{1}(0)-i}{\tilde{\alpha}_{1}(0)+i} \cdot \frac{\tilde{\beta}_{1}(0)-i}{\beta_{1}(0)+i}\right|<1
$$

We note however that unless either $\tilde{\alpha}_{1}(0)$ or $\tilde{\beta}_{1}(0)$ is complex this inequality cannot be satisfied. Otherwise, if both $\tilde{\alpha}_{1}$ and $\tilde{\beta}_{1}$ are real the absolute value of each ratio (and hence the product) is one. We conclude from this that the correctly posed boundary value problem for an elliptic partial differential equation must be given in terms of complex valued functions $\tilde{\alpha}_{0}(x), \tilde{\alpha}_{1}(x), \tilde{g}(x)$ of the real variable $x$, and complex valued functions $\tilde{\beta}_{0}(y), \tilde{\beta}_{1}(y), \tilde{h}(y)$ of the real variable $y$.

The uniqueness theorem we have proved then is for complex valued solutiuns $u(x, y)=u^{(1)}(x, y)+i u^{(2)}(x(y)$ of the elliptic partial differential equation

$$
\Delta u=\tilde{f}\left(x, y, u, u_{x}, u_{y}\right)
$$

which satisfy the four generalized boundary value conditions $(1.4 \mathrm{a}, \mathrm{b}),(1.5 \mathrm{a}, \mathrm{b})$, and the intersection condition $(1.5 \mathrm{c})$. 
The condition that data cannot be given on two straight lines may be seen to be an unnecessary one. It may be seen using VEKUA's theory of generalized analytic functions in conjunction with our approach that for the case of the problem discussed here (1.1), (1.2), (1.3) data can indeed be given on two straight lines. It appears therefore that the difficulty in treating the case of data on two stright lines is an artificial one due to the particular manner in which we introduced the various operators. These results will be presented in a future paper.

Acknowlodgement. - We should like to thank Professor LIXCOLN BRAGG for interesting and useful discussions concerning this research problem, which have aided in the preparation of this manuscript, and Mr. Brevster Knight for carefully reading the manuscript and making several suggestions which have improved the presentation.

\section{REFERENCES}

A. 1 A.K. Azız, On a functional integral equation with applications to hyperbolic partial differential equations, Duke Math. Journal, Vol. 32, No, 4, pp. 579.592 (1965).

A.B. 1 A.K. AzIz and A. BogDonowcz, A generalizrd Goursat problem for non-linear hyperbolic partial differential equations, (to appear).

A.G. 1 A.K. Aziz and R.P. GILBkrT, A genaralized Goursat problem for elliptic equations, die Journal für Reine and Angewandte Mathematik Band 222, Heft 1/2, pp. 1-13 (1966).

B. 1 S. Bergman, Integral operators in the theory of linear partial differential equations, Ergeb. Math. u. Grenzeb, 2s, Springer, Berlin (1960).

B.S. 1 S. Bergmar and M. SommFer, Kernel functions and elliptic differential equations in mathematical physics, Academic Press, New York (195̃3).

F. 1 B.A. FuKs, Introduction to the theory of analytic functions of several complex variables, Translations of Mathematical Monographs, 8 Amer. Math. Soc., Providence, (1963).

H. 1 P. HENRICr, A survey of I.N. Veleua's Theory of elliptic partial differential equations with analytic coefficients, J. Applied Math. Phisics, (ZAMP), Vol. VIII, No. 3 (2957) pp. $169-203$.

H. 2 E. HrLe, Analytic Function Theory, Vol 1, Ginn and Co., Boston (1959).

K. 1 J. KISYNSKI, Sur l'esxistence des solutions d'un probleme de Z. Szmydt relativ a l'equation $\frac{\partial^{2} u}{\partial x \partial y}=f\left(x, y, u, u_{x}, y y\right)$, Ann. Univ. M. Curie Sklodowska, Sec. A, 12 (1958), pp. 67.109 .

K.F. 1 A.N. KommogoroF and S.V. Fomis, Elements of the theory of functions and functional analysis, Vol. 1, Graylock Press, Rochester (1951).

S. 1 Z. Szmyor, Sur une generalization des problemes classiques concernant un system d'equation differentielles hyperbolic du secon ordre a deux variables independentes, Bull. Acad. Polon. Sci. Cl. 1II, 4 (1956).

V. 1 I.N. VEKUA, Novye metody rešnija elliptcěskikh uravnenij. (Now methods for solving elliptic equations), (OGIZ) Moskow and Leningrad (1948).

V. 2 - Generalized analytic functions, Addison-Wesley, Reading (1962). 